\title{
Satisfação com a imagem corporal em mulheres gestantes e não gestantes
}

\section{Body image satisfaction in pregnant and non-pregnant woman}

Fabiano Augusto Teixeira ${ }^{1}$, Voldiana Lúcia Pozzebon Schneider², Raquel Eleine Wolpe ${ }^{1}$, Fabiana Flores Sperandio

${ }^{1}$ Laboratório de Saúde da Mulher do Centro de Ciências da Saúde e do Esporte da Universidade do Estado de Santa Catarina (UDESC) - Santa Catarina (SC), Brasil.

${ }^{2}$ Curso de Fisioterapia da UDESC - Santa Catarina (SC), Brasil.

DOI: http://dx.doi.org/10.7322/abcshs.v40i2.733

\section{RESUMO}

Introdução: A imagem corporal (IC) associa-se ao autoconceito com foco na aparência e na forma corporal, entendida como a representação mental do próprio corpo como um processo dinâmico e singular. Objetivo: Este estudo objetivou analisar a percepção da IC de mulheres gestantes e compará-las com um grupo de não gestantes. Métodos: Utilizou-se a Escala de Nove Silhuetas. Fizeram parte do estudo 60 mulheres, divididas em 4 grupos com 15 participantes cada, sendo os grupos 1, 2 e 3 compostos por mulheres com idade gestacional (IG) de $16^{\mathrm{a}}, 26^{\mathrm{a}}$ e $36^{\mathrm{a}}$ semanas, respectivamente, contadas a partir da data da última menstruação, e um grupo de não gestantes. Os dados foram tabulados e analisados no programa estatístico SPSS ${ }^{\circledR}$. Resultados: A maioria das mulheres participantes do estudo acredita que a silhueta ideal é a mais magra do que a silhueta atual. O índice de massa corporal (IMC) foi diferente para todos os grupos pesquisados, indicando um aumento no peso com o decorrer da gestação. Com isso, percebeu-se que as gestantes apresentam uma forma singular de observar as mudanças no seu corpo de acordo com a IG em que se encontram e com as experiências vividas anteriormente. Com relação à diferença na percepção corporal de gestantes e não gestantes, não foi possível observar diferenças significativas entre os grupos. Conclusão: Existe a necessidade de elaborar um instrumento de pesquisa que represente as gestantes, tanto na posição anatômica quanto na posição de perfil, nas diferentes semanas gestacionais, para que a visualização da forma corporal seja mais adequada à realidade física das gestantes.

Palavras-chave: gestantes; imagem corporal; bem-estar materno.

\begin{abstract}
Introduction: The body image is associated with the selfconcept with focus on appearance and body shape, understood as the mental representation of the body as a dynamic and unique process. Objective: This study aimed to analyze the perception of body image of pregnant women and compare them with a group of non-pregnant women. Methods: We used the Scale nine silhouettes. Study participants were 60 women divided into 4 groups with 15 participants each, with group 1, 2 and 3 with a gestational age of 16th, 26th and 36th weeks, respectively, counted from the date of last period and a group with non-pregnant women. Data was tabulated and analyzed in SPSS statistical program. Results: The majority of women participating in this study, in both groups believe that the ideal silhouette is slimmer than the current one. Body mass index (BMI) was different for all groups surveyed, indicating an increase in weight over the course of pregnancy. Thus, it was noticed that pregnant women have a unique way of observing the changes in their bodies according to gestational age in which they are and with previous experiences. Regarding the difference in body perception among pregnant and nonpregnant women there was no significant observed between the groups. Conclusion: There is a need to design up a research tool, representing pregnant women both in the anatomical position, as in the profile position in the different gestational weeks, for the visualization of body shape is more appropriate to the physical reality of pregnant women.
\end{abstract}

Keywords: pregnant women; body image; maternal welfare. 


\section{INTRODUÇÃO}

A gestação é especial na vida da mulher; embora possa ser considerada um período conturbado, no qual ocorrem diversas mudanças físicas e alterações substanciais no seu peso e na sua forma corporal ${ }^{1}$, tal fase é caracterizada por transformações corporais relacionadas aos ritmos metabólicos e hormonais, demandando o processo de integração de uma nova imagem corporal (IC) $)^{2}$.

A IC pode ser entendida como a representação mental do próprio corpo, sendo dinâmica e singular; é um constructo multifacetado constituído por componentes atitudinais e perceptivos - emocionais, cognitivos e comportamentais - que se estabelecem em um continuum $^{3}$. Tais elementos partem de valores positivos e usualmente associados a sentimentos e comportamentos saudáveis, tais como a prática de exercícios, os cuidados com o corpo, as relações sociais estáveis e a autoestima. Para valores negativos, tem-se a associação de outros quadros clínicos, como a depressão e a obesidade $^{4}$. Desse modo, a IC é um fenômeno complexo que deve ser visto sob múltiplas perspectivas e engloba todas as formas pelas quais a pessoa vivencia e conceitua seu próprio corpo ${ }^{3}$.

Durante a gestação ocorrem alterações naturais ao corpo da mulher, como, por exemplo, o ganho de peso; consequentemente, muitas mulheres apresentam dificuldades em aceitá-las, tornando-se inseguras e mais propensas a ter preocupação com sua IC $^{5}$. Nesse sentido, a gestação está inserida em um contexto único e complexo que comporta não somente a história pessoal da mulher grávida, sua sexualidade, seu passado ginecológico e obstétrico e seus desejos, mas também o momento presente, não existindo um padrão ideal de gravidez $z^{6}$

Os sentimentos durante a gravidez são mistos e interagem dinamicamente no decorrer de aproximadamente 42 semanas determinando o contexto emocional da gravidez. Essa diversidade pode ser observada na mulher ao curso de diferentes gestações ou mesmo em uma única gravidez ${ }^{7}$.

Partindo do exposto e considerando que são escassos os estudos na literatura que avaliam a temática IC em gestantes, este estudo teve como objetivo analisar a percepção da IC de mulheres gestantes e, por fim, compará-las com um grupo de não gestantes, a fim de oferecer informações para profissionais da saúde, especialistas e não especialistas, ao passo que ambos lidam diretamente com esse público e carecem de informações acerca da temática a ser investigada neste estudo.

\section{MÉTODOS}

O presente estudo obteve a aprovação do Comitê de Ética em Pesquisa em Seres Humanos da Universidade do Estado de Santa Catarina (UDESC).

Este estudo transversal se caracteriza como descritivo e quantitativo. A etapa descritiva visa descrever, registrar, interpretar e correlacionar fatos ou fenômenos não manipuláveis, isto é, procura descobrir, com a precisão possível, a frequência com que o fenômeno ocorre, sua relação e sua conexão com outros fenômenos, sua natureza e suas características ${ }^{8}$. Por fim, a etapa quantitativa permite que sejam realizadas análises numéricas dos dados levantados pelos instrumentos utilizados, sendo capazes de estabelecer relações e causas, levando em conta as quantidades ${ }^{9}$.

O recrutamento das gestantes deu-se em uma clínica particular de ginecologia e obstetrícia, com participantes em acompanhamento pré-natal, na qual as participantes aguardavam sua consulta na sala de espera. Essas foram abordadas pelos pesquisadores do estudo e, após explicados os objetivos da pesquisa, foram convidadas a participar. Todas as participantes que aceitaram participar do estudo são maiores de 18 anos e assinaram o Termo de Consentimento Livre e Esclarecido, enquanto a abordagem das mulheres não gestantes para este estudo ocorreu com universitárias em uma instituição pública de ensino.

Para este estudo, foi adotado como critérios de inclusão estar em idade gestacional (IG) em 16a , 26 e 36 a semanas, referindo-se respectivamente ao $1^{\circ}, 2^{\circ}$ e $3^{\circ}$ trimestres de gestação. Os critérios de exclusão foram diagnóstico prévio de hipertensão, diabetes ou anemia.

Inicialmente, os dados foram coletados com 88 mulheres, porém 28 foram excluídas por não atenderem aos critérios de inclusão do estudo.

Assim, fizeram parte da amostra 60 mulheres, divididas em quatro grupos com 15 participantes cada, sendo o grupo 1 (G1) formado por mulheres na $16^{\mathrm{a}}$ semana de gestação, o grupo 2 (G2), na $26^{\mathrm{a}}$ semana, o grupo 3 (G3), na $36^{\mathrm{a}}$ semana, e, por fim, o grupo 4 (G4), com mulheres não gestantes. Destaca-se que este estudo considerou as semanas de gestação contadas a partir da data da última menstruação (DUM) relatada pelas participantes, aceitando-se variação de uma semana para mais ou para menos.

Para a coleta das informações, utilizou-se um questionário contendo questões acerca da caracterização das participantes (grau de escolaridade, idade em anos, peso em kg, altura em cm e ganho de peso pós-gestacional). A massa corporal e a estatura foram obtidas por meio de medidas autorreferidas. A validade dessas medidas tem sido comprovada com a população adulta brasileira ${ }^{10}$. Para classificação do estado nutricional da gestante, foram utilizados os pontos de corte estabelecidos pelo Ministério da Saúde ${ }^{11}$ o qual leva em consideração a aferição do peso e da estatura da mulher e o cálculo da IG.

Para avaliar a percepção da IC, utilizou-se a Escala de Desenhos de Nove Silhuetas, a qual apresenta uma sequência de figuras de imagens corporais com nove variações em ordem crescente de tamanho corporal $^{12}$. Neste caso, a participante deveria apontar: 1) aquela figura que melhor a representava no momento atual e 2) apontar, posteriormente, a silhueta que representasse o seu ideal. Feito isso, o nível de satisfação ou insatisfação com a IC foi obtido por meio da subtração da autopercepção da IC atual pela autopercepção da imagem ideal. Os valores poderiam variar de $-8 \mathrm{a}+8$, quais sejam: $-8 \mathrm{a}-1$ (insatisfeito por magreza), 0 (satisfeito) e, por fim, 1 a 8 (insatisfeito por excesso de peso). Ao final do instrumento questionou-se a autopercepção da silhueta corporal que representasse a IC antes da gestação.

Para caracterização da amostra, foi empregada a análise descritiva por meio dos valores de frequência absoluta e relativa, e, no 
caso das variáveis contínuas, pelo cálculo de média, desvio padrão e mediana. Ao verificar a não normalidade dos dados, as variáveis foram comparadas entre os grupos pelo teste de Kruskal-Wallis. Para o estudo entre as proporções das variáveis IC atual, IC após o parto e IC ideal, utilizou-se o teste do $\chi^{2}$, adotando um nível de significância de $\alpha<0,05$. Os dados foram tabulados e analisados com o auxílio do programa estatístico Statistical Package for the Social Sciences (SPSS) ${ }^{\circ}$ for Windows, versão 20.0.

A caracterização geral das participantes pode ser observada na Tabela 1.

\section{RESULTADOS}

A Tabela 2 apresenta os valores obtidos em média e desvio padrão das participantes quanto a idade, peso, ganho de peso e índice de massa corporal (IMC). Os itens idade e peso não foram diferentes do ponto de vista estatístico ( $p>0,05)$.

A variação do IMC obteve resultado estatisticamente significativo para todos os grupos, com p=0,002 (Tabela 2).

Pode-se observar (Tabela 3) que para o ganho de peso houve diferença estatisticamente significativa $(p<0,05)$ entre o $G 1$ e o G2 e entre o G1 e o G3, mas não houve diferença significativa ( $p>0,05)$ entre o G2 e o G3. Desse modo, esses valores apontam que houve aumento de peso significativo no início da gestação,

Tabela 1: Caracterização das participantes do estudo

\begin{tabular}{|c|c|c|c|c|}
\hline \multirow[t]{2}{*}{ Variável } & $\begin{array}{c}\text { G1 } \\
16 \\
\text { semanas } \\
(n=15)\end{array}$ & $\begin{array}{c}\text { G2 } \\
26 \\
\text { semanas } \\
(n=15)\end{array}$ & $\begin{array}{c}\text { G3 } \\
36 \\
\text { semanas } \\
(n=15)\end{array}$ & $\begin{array}{c}\text { G4 } \\
\text { Não } \\
\text { gestantes } \\
(n=15)\end{array}$ \\
\hline & n (\%) & n (\%) & n (\%) & n (\%) \\
\hline \multicolumn{5}{|c|}{ Grau de escolaridade } \\
\hline $\begin{array}{l}\text { Até } 1^{\circ} \text { grau } \\
\text { completo }\end{array}$ & $2(13,3)$ & $3(20)$ & $1(6,6)$ & $0(0)$ \\
\hline $\begin{array}{l}\text { Até } 2^{\circ} \text { grau } \\
\text { completo }\end{array}$ & $8(53,3)$ & $9(60)$ & $10(66,6)$ & $4(26,6)$ \\
\hline $\begin{array}{l}\text { Até } 3^{\circ} \text { grau } \\
\text { completo }\end{array}$ & $5(33,3)$ & $3(20)$ & $4(26,6)$ & $11(73,3)$ \\
\hline \multicolumn{5}{|c|}{ Idade (em anos) } \\
\hline Até 20 & $0(0)$ & $1(6,6)$ & $2(13,3)$ & $1(6,6)$ \\
\hline $20-29$ & $11(73,3)$ & $11(73,3)$ & $11(73,3)$ & $13(86,6)$ \\
\hline $30-39$ & $4(26,6)$ & $3(20)$ & $2(13,3)$ & $1(6,6)$ \\
\hline
\end{tabular}

Tabela 2: Idade, peso, ganho de peso e índice de massa corporal das participantes do estudo

\begin{tabular}{|c|c|c|c|c|}
\hline Variável* & $\begin{array}{c}\text { G1 } \\
(n=15) \\
\text { Média (DP) }\end{array}$ & $\begin{array}{c}\text { G2 } \\
(n=15) \\
\text { Média (DP) }\end{array}$ & $\begin{array}{c}\text { G3 } \\
(n=15) \\
\text { Média (DP) }\end{array}$ & $\begin{array}{c}\text { G4 } \\
(n=15) \\
\text { Média (DP) }\end{array}$ \\
\hline $\begin{array}{l}\text { Idade (em } \\
\text { anos) }\end{array}$ & $27,6(4,97)$ & $25,7(3,88)$ & $24,0(4,38)$ & $24,0(3,1)$ \\
\hline Peso (em kg) & $61,5(12,22)$ & $69,2(9,7)$ & $68,7(8,04)$ & $62,8(8,29)$ \\
\hline $\begin{array}{l}\text { Ganho de } \\
\text { peso (em kg) }\end{array}$ & $3,9(2,26)$ & $8,89(4,29)$ & $11,19(4,48)$ & - \\
\hline IMC & $23,9(4,7)$ & $26,1(2,75)$ & $26,7(3,12)$ & $22,2(2,87)$ \\
\hline
\end{tabular}

*Valores expressos como médias e desvio padrão

dp: desvio padrão; IMC: índice de massa corporal representado pelas diferenças entre o G1 e o G2 e entre o G1 e o G3. Porém o aumento de peso no final da gestação não foi muito elevado e, consequentemente, não houve diferença estatística significativa entre o G2 e o G3.

Ao observar a Tabela 3 , nota-se que o valor do $\chi^{2}$ para o G1 versus o $\mathrm{G} 4$ foi de 18,92, para o G2 versus o G4, de 5,62, e, por fim, para o G3 versus o G4, de 11,23. E ainda, G1, G2 e G3, quando comparados ao G4, apontaram diferença estatisticamente significativa, ilustrando ganho de peso entre os grupos.

Ao comparar a silhueta ideal com a silhueta atual, observamos que praticamente todas as mulheres consideram que a silhueta ideal é menor do que a sua silhueta atual (Figura 1).

Para investigar o aumento corporal com o avanço da gravidez, subtraiu-se a silhueta corporal atual daquela que representava o corpo da participante no momento pré-gestacional. As variações das silhuetas são apresentadas na Figura 2.

Para investigar a expectativa das mulheres com relação ao seu corpo logo após o nascimento do bebê, subtraiu-se, igualmente, a silhueta ideal para o período pós-parto da silhueta que a representava durante a gestação. Os resultados são apresentados na Figura 3.

Tabela 3: Índice de significância para o ganho de peso entre diferentes grupos de gestantes e não gestantes

\begin{tabular}{|c|c|c|}
\hline Gestantes versus G4 & $\chi^{2}$ & Valor $p$ \\
\hline G1 & 18,92 & $<0,000078$ \\
\hline G2 & 5,62 & $<0,058$ \\
\hline G3 & 11,23 & $<0,0036$ \\
\hline Grupo de gestantes & \multicolumn{2}{|c|}{ Valor $p$} \\
\hline Entre G1 e G2 & \multicolumn{2}{|c|}{$<0,001$} \\
\hline Entre $\mathrm{G} 1$ e $\mathrm{G} 3$ & \multicolumn{2}{|c|}{$<0,001$} \\
\hline Entre G2 e G3 & \multicolumn{2}{|c|}{$<0,179$} \\
\hline
\end{tabular}

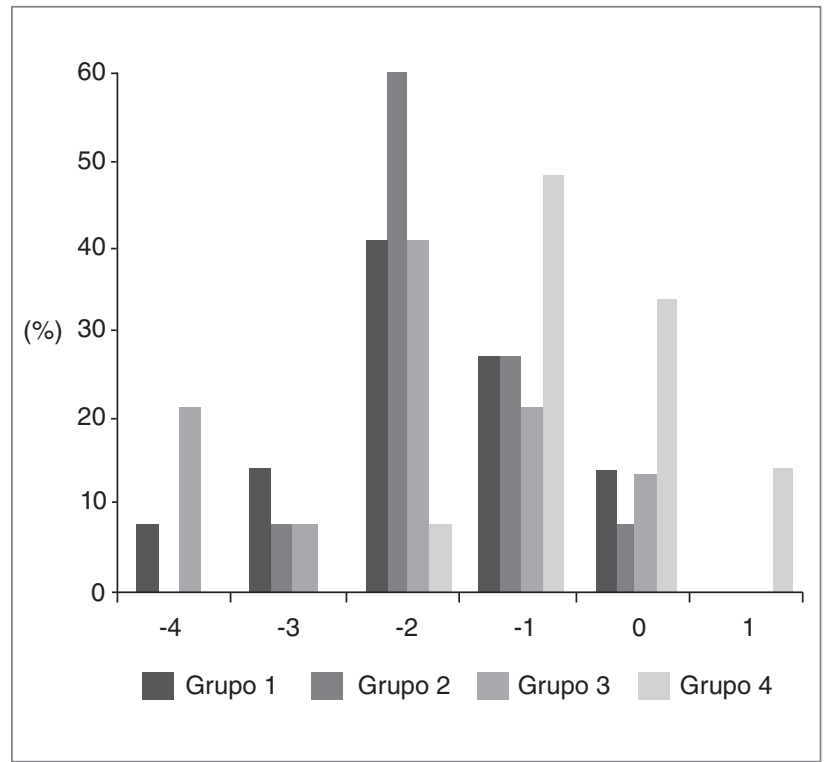

Figura 1: Valores que representam a imagem ideal subtraída da imagem atual 


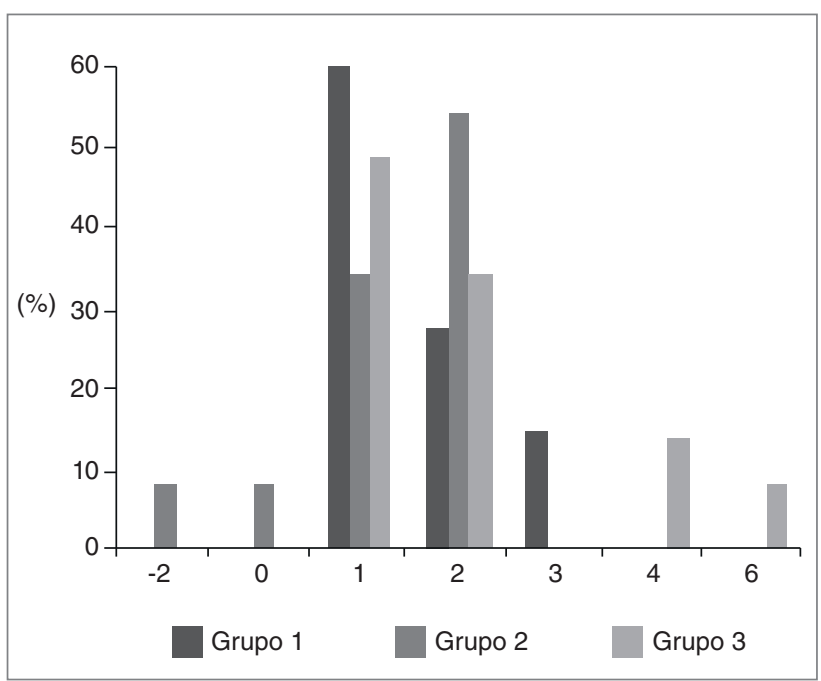

Figura 2: Variação da silhueta atual subtraída da silhueta corporal pré-gestacional

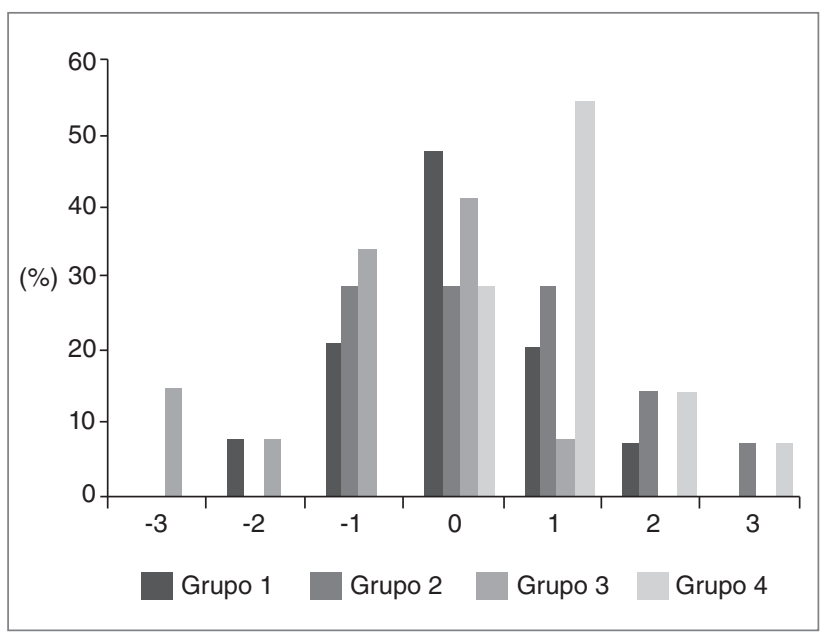

Figura 3: Variação da silhueta corporal pós-gestacional subtraída da silhueta atual

\section{DISCUSSÃO}

Durante nove meses a mulher passa por inúmeras transformações ao conciliar o papel de gestante e mulher na sociedade. Nesse período, surgem muitas dúvidas e questionamentos pertinentes ao estado físico e emocional da gestante, entre eles a satisfação ou a insatisfação com a IC advinda das semanas em que decorre a gestação. Muitos aspectos da gestação continuam sendo explorados ou até mesmo inadequadamente discutidos por diferentes profissionais de saúde.

O ganho de peso recomendado durante a gestação varia conforme a estrutura corporal da mulher antes de engravidar. Mulheres com peso adequado antes da gestação devem ter um ganho entre 8,5 a $14,5 \mathrm{~kg}^{13}$. Para mulheres que estavam abaixo do peso antes de engravidar, a literatura recomenda um ganho entre 10,5 a 18,0 kg; no entanto, para mulheres com peso acima do recomendado antes da gestação, o ganho deve ficar entre 5,5 a 10,2 $\mathrm{kg}^{14}$.
De acordo com estudos anteriores ${ }^{15,16}$, percebe-se que as gestantes apresentaram uma forma singular de observar as mudanças no seu corpo, de acordo a IG em que se encontram e com as experiências vividas anteriormente. Com relação às formas pré-gestacionais e gestacionais, as mulheres sugeriram aumento de uma ou duas silhuetas corporais, independentemente da IG. As gestantes do G1 (IG=16 semanas) perceberam o seu corpo de forma similar às gestantes do G3 (IG=36 semanas), enquanto as gestantes do G2 ( $\mathrm{IG}=26$ semanas) perceberam alterações maiores na forma corporal do que as do G3. A similaridade entre o G1 e o G3 pode ser explicada pela ansiedade do G1 em ver o corpo aumentar para confirmar a gestação, fazendo com que no início da gravidez a percepção das alterações corporais seja maior do que o real ${ }^{17}$.

No entanto, no final da gravidez as mulheres já vivenciaram praticamente todas as mudanças físicas da gestação e percebem que as alterações corporais podem não ser tão significativas ${ }^{18}$. Ou então, como o seu corpo está em constante mudança, elas perdem o referencial da sua forma corporal pré-gestacional e não identificam a real diferença entre os dois períodos ${ }^{12}$.

Quanto à projeção da imagem física após o parto, há uma expectativa de manter a silhueta atual, independentemente da IG.

Com relação à silhueta ideal, as que se encontram à esquerda da escala de avaliação foram as mais apontadas, tendendo para um corpo magro e de curvas definidas. É necessário atentar para a desigualdade entre a imagem ideal e a imagem atual encontradas neste estudo. Tais descontentamentos com a forma física podem ter influência negativa na autoestima das gestantes durante a gravidez, gerando exigências internas não elucidadas. Os estudos que investigam essa questão são em número reduzido, e a discussão contínua de sinais de angústias e sofrimentos com a forma física pode favorecer o manejo dos diferentes profissionais envolvidos com a satisfação e o bem-estar desse coletivo ${ }^{15,16}$.

Com relação à imagem corporal ideal, observou-se um percentual de respostas mais elevado nas silhuetas situadas à esquerda da escala, ratificando o fato de elas acreditarem que a silhueta ideal é a mais magra. Em um estudo realizado com 237 mulheres gestantes e não gestantes se identificou similaridade à insatisfação corporal, ao passo que os pesquisadores ilustraram o relato de mulheres insatisfeitas com a imagem corporal por excesso (76\%), acreditando, igualmente, que a silhueta ideal é a mais magra ${ }^{19}$. E ainda, quando se comparou a silhueta ideal com a silhueta atual, observou-se que praticamente todas as mulheres consideram que a silhueta ideal é menor do que a sua atual (Figura 2). Isso reflete a tendência atual de as mulheres não se satisfazerem com a imagem que seus corpos apresentam ${ }^{15}$ e uma possível distorção de tal aparência.

A maioria das gestantes referiu aumento da forma corporal (números positivos da Figura 2). Enquanto no G2, além do aumento, houve também a manutenção (número zero) e a diminuição da silhueta corporal (números negativos), simultaneamente, o aumento corporal tanto no início da gestação (G1) quanto ao final (G3) correspondeu, em média, a uma ou duas silhuetas. Esse fato chama a atenção, pois, embora existam 
muitas mudanças entre os dois períodos gestacionais, as participantes identificam praticamente as mesmas alterações na silhueta corporal. De outro lado, estudo ${ }^{20}$ ilustra a autopercepção de gestantes quanto à imagem corporal, as quais identificaram discrepâncias na silhueta corporal.

De uma maneira geral, as mulheres esperam uma variação pequena em seu corpo após o parto, aumentando ou diminuindo apenas uma silhueta. Esse fato é interessante, pois, apesar de haver nítida diferença entre a $16^{\mathrm{a}}$ e a $36^{\mathrm{a}}$ semana gestacional, as mulheres esperam manter após o parto a silhueta da semana gestacional que relataram no início da gestação. Pesquisas ${ }^{21,22}$ apontam que, para as mulheres conseguirem manter a diminuição da silhueta corporal após o parto, faz-se necessário que pratiquem atividade física com regularidade, sob a supervisão de um profissional da área.

Conclui-se, ao analisar a percepção da imagem corporal de mulheres gestantes e compará-las com um grupo de não gestantes, que as mulheres sugeriram um aumento de uma ou duas silhuetas corporais, independentemente da IG. As gestantes com 16 semanas gestacionais identificaram o seu corpo de forma similar às gestantes com 36 semanas, diferentemente das mulheres com 26 semanas, em que as alterações na forma corporal foram maiores. Com relação à diferença na percepção corporal entre gestantes e não gestantes, não foi possível observar alterações significativas entre os grupos.

Sugere-se que estudos longitudinais sejam realizados com gestantes, a fim de verificar as alterações da satisfação com a imagem corporal com o passar dos diferentes trimestres. E ainda, este estudo se limitou a utilizar uma escala de silhuetas validada para a população adulta feminina não gestante em uma amostra de gestante; com isso, a partir dos resultados encontrados neste estudo, verificou-se que se faz necessário que os pesquisadores elaborem novas pesquisas com o intuito de elaborar um instrumento que represente as gestantes no plano frontal e sagital, para a visualização da forma corporal seja mais adequada à sua realidade física.

Por fim, outras pesquisas são necessárias para melhor compreensão dos fatores que podem influenciar a satisfação ou a insatisfação com a imagem corporal, como estudos com abordagem qualitativa, que podem aprofundar os conhecimentos acerca do tema.

\section{REFERÊNCIAS}

1. Bell R, Palma S. Antenatal exercise and birthweight. Aust N Z J Obstet Gynaecol. 2000;40(1):70-3.

http://dx.doi.org/10.1111/j.1479-828X.2000.tb03171.x

2. Oliboni CM, Galletta MAK, Francisco RPV, Alvarenga MS. Adaptação transcultural da Escala de Atitudes em Relação ao Ganho de Peso na Gestação Cross-cultural adaptation of the Pregnancy and Weight Gain Attitude Scale. Rev Bras Ginecol Obstet. 2014;36(7):320-27.

http://dx.doi.org/10.1590/SO100-720320140004998

3. Gonçalves CO, Campana NA, Tavares MC. Influência da atividade física na imagem corporal: uma revisão bibliográfica. Motri. 2012;8(2):70-82.

http://dx.doi.org/10.6063/motricidade.8(2).716

4. Pelegrini A, Coqueiro RS, Beck CC, Ghedin KD, Lopes AS, Petroski EL. Dissatisfaction with body image among adolescent students: association with socio-demographic factors and nutritional status. Ciênc Saúde Coletiva. 2014;19(4):1201-8. http://dx.doi.org/10.1590/1413-81232014194.09092012

5. Clark A, Skouteris H, Wertheim EH, Paxton SJ, Milgrom J. The relationship between depression and body dissatisfaction across pregnancy and the postpartum: a prospective study. J Health Psychol. 2009;14(1):27-35.

http://dx.doi.org/10.1177/1359105308097940

6. Szejer M, Stewart R. Nove meses na vida da mulher: uma abordagem psicanalítica da gravidez e do nascimento. São Paulo: Casa do Psicólogo; 1997. p.322.

7. Richardson P. Women's experiences of body change during normal pregnancy. Matern Child Nurs J. 1990;19(2):93-111.

8. Baruffi H. Metodologia Científica. Manual para elaboração de monografias, dissertações, projetos e relatórios de pesquisas. Dourados: HB Edit; 1998.
9. Ribeiro JLD, Echeveste MÊS, Danilevicz AM de. A utilização do QFD na otimização de produtos, processos e serviços. Porto Alegre: FEEng/UFRGS; 2001.

10. Coqueiro RS, Borges LJ, Araújo VC, Pelegrini A, Barbosa AR. Medidas auto-referidas são validas para avaliação do estado nutricional brasileira? Rev Bras Cineantropom Desempenho Hum. 2009;11(1):113-19.

11. Brasil. Ministério da Saúde. Secretaria de Atenção à Saúde. Departamento de Atenção Básica. Obesidade. Série A. Normas e Manuais Técnicos. Brasília: Ministério da Saúde; 2006.

12. Stunkard AJ, Sorensen T, Schulsinger F. Use of the danish adoption register for the study of obesity and thinness. In: Kety SS, Rowland LP, Sidman RL, Matthysse, SW. The genetics of neurological and psychiatric disorders. New York: Raven Press; 1983. p. 115-20.

13. Drehmer M. Ganho de peso gestacional, desfechos adversos da gravidez e retenção de peso pós-parto. Tese (Doutorado) Programa de Pós-Graduação em Epidemiologia, Faculdade de Medicina da Universidade Federal do Rio Grande do Sul, Porto Alegre, 2010.

14. Park S, Sappenfielg WM, Bish C, Salihu H, Goodman D, Bensyl $D$. Assessment of the Institute of Medicine recommendations for weight gain during pregnacy: Florida 2004-2007. Matern Child Health J. 2011;15(3):289-301.

15. Ghosh A. Anthropometric and body composition characteristics during pregnancy: a stydy from West Bengal India. Homo. 2012;63(3):233-40.

16. Drehmer M, Duncan BB, Kac G, Schmidt MI. Association of second and third trimester weight gain in pregnancy whit maternal and fetal outcomes. PLoS One. 2013;8(1):e54704.

http://dx.doi.org/10.1371/journal.pone.0054704 
17. Sui Z, Dodd JM. Exercise in obese pregnant women: positive impacts and current perceptions. Int $J$ Womens Health. 2013;5:389-98. http://dx.doi.org/10.2147/IJWH.S34042

18. Ko CW, Napolitano PG, Lee SP, Schulte SD, Ciol A, Beresford SA. Physical activity, maternal metabolic measures, and the incidence of gallbladder sludge or stones during pregnancy: a randomized trial. Am J Perinatol. 2014;31(1):39-48. http://dx.doi.org/10.1055/s-0033-1334455

19. Passanha A, Martins APB, Silva ACF, Benécio MHD. Insatisfação com a imagem corporal no período pré-gestacional e fatores associados. Rev Psicol Saúde. 2013;5(2):92-101.
20. Nobre RN, Meireles AVP, Frota JT, Costa RMM, Coutinho VF, Garcia MMCM, et al. Comportamento alimentar e percepção da imagem corporal de gestantes atendidas em um ambulatório de alto risco. Rev Bras Promoção Saúde. 2014;27(2):256-62. http://dx.doi.org/10.5020/18061230.2014.p256

21. Sui Z, Turnbull D, Dodd J. Effect of body image on gestational weight gain in overweight and obese women. Women Birth. 2013;26(4):267-72

22. Loth KA, Bauer KW, Wall M, Berge J, Neumark-Sztainer D. Body satisfaction during pregnancy. Body Image. 2011;8(3):297-300. 